\title{
Oral Cancer Knowledge, Attitudes and Practices among Primary Oral Health Care Dentists in Kuwait
}

\author{
Huda Nazar ${ }^{1 *}$, Maddi Shyama ${ }^{2}$, Jitendra Ariga ${ }^{3}$, Mohamed El-Salhy ${ }^{4}$, Pramod \\ Soparkar ${ }^{5}$, Aishah Alsumait ${ }^{2}$
}

\begin{abstract}
Background: Oral cancer signifies a public health concern of international importance. Early detection of oral cancer can improve the prognosis and the 5-year survival rate. Objective: The aim of this study was to assess the level of oral cancer knowledge, opinion, attitudes and practices among dentists working at the primary oral health care centers in Kuwait. Methods: In this cross-sectional study, self-reported questionnaire was distributed to the dentists working at the primary oral health care centers in Kuwait. A total of 289 dentists participated in the present study. The questionnaire included 23- questions on oral cancer knowledge, opinion, attitudes and practices. Results: The mean age of the dentists was $35.2 \pm 10.9$ years. Approximately, all dentists (99.7\%) were aware of the major risk factors that were most likely associated with oral cancer. Overall, majority of the participants knew the most common form of oral cancer $(80.6 \%)$, most common site $(80.3 \%)$ and the likely lesions associated with oral cancer occurrence $(87.9 \%)$. A large number of dental practitioners $(81 \%)$ would routinely refer a patient with a suspicious lesion to a specialist. Nearly one-third (32\%) reviewed their patients' oral cancer risk factors. Approximately two-thirds $(62 \%)$ assessed the use of tobacco in their practice. Almost, all (92.4\%) were interested in attending continuing education courses on oral cancer. Conclusions: Majority of the participants presented good knowledge about various aspects of oral cancer. More continuing education programs on risk factors and diagnosis of oral cancer should be organized to train the dentists. Oral cancer screening should be a routine procedure for the high risk patients at the primary oral health care centers in Kuwait.
\end{abstract}

Keywords: Oral cancer- knowledge- primary oral health care dentists- Kuwait

Asian Pac J Cancer Prev, 20 (5), 1531-1536

\section{Introduction}

Oral and oropharyngeal cancer is a major part of the global burden of cancer and is the sixth most common cancer in the world (Warnakulasuriya, 2009), with increasing rates of incidence and rising mortality rates (Gillison, 2007; Petersen, 2009; Kujan et al., 2017). Late diagnosis, high mortality rates and morbidity leading to important disfigurement are characteristics of the disease worldwide. Oral cancer is a universal health problem and is more common in the developing countries than in the developed countries (Thun et al., 2010; Petersen, 2009; Kujan et al., 2017).

The major risk factors attributed to oral cancer occurrence are the use of tobacco; chewing, snuffing, or smoking and the consumption of alcohol (Moreno-Lopez et al., 2000; Rodriguez et al., 2004; Petersen, 2009). Also, in a systematic review, it was found that there is an association between socioeconomic status and oral cancer prevalence (Conway et al., 2008). In 2007, the World Health Organizations (WHO's) global policy on oral health, which also considered oral cancer prevention, emphasized that the involvement of oral health professionals in early detection, diagnosis and treatment as an integral part of national cancer control programmes (Petersen, 2008).

In Kuwait, head and neck cancers are common sites of occurrence, the most predominant site being the nasopharynx (Morris et al., 2000). In an earlier study, Morris et al., (2000) conducted a computer search at the Kuwait cancer registry, for all cancers of the lip, oral cavity, and pharynx in the 10 years, from 1979 to 1988 . They recorded 784 cases $(7.4 \%)$ during this period out of a total of 10,539 cancers; the most frequent sites of cancer were the nasopharynx (25\%), salivary glands $(24 \%)$, and hypopharynx (14\%). In 2008, the oral cancer incidence rate in Kuwait was 1.4 (per 100,000) and the mortality rate 0.6 . The incidence rate in women was higher (1.8 per 100,000) than in men (1.1) (Ferlay et al., 2008). The male: female ratio for both incidence and mortality rates in 2012 were 1.5:1.3 and 0.3:0.7 respectively (Ferlay et al., 2012). Although the mortality rate is not very high in Kuwait, early detection of oral cancer is needed because

${ }^{1}$ Research and Survey Division, Dental Administration, ${ }^{2}$ National Oral Health Program, Ministry of Health, ${ }^{3}$ School Oral Health Program, Kuwait-Forsyth, Kuwait, ${ }^{4}$ College of Dental Medicine, University of New England, Portland, Maine, ${ }^{5}$ Forsyth Institute, Cambridge, MA, USA.*For Correspondence: hudanazar@yahoo.com 
of the increased consumption of tobacco in the last few years among Kuwaiti population (Memon et al., 2000).

Early detection of oral cancer can improve the prognosis and the 5-year survival rate (Yellowitz et al., 2000; Messadi et al., 2009). Although most of the dentists mentioned that they perform an oral examination on their patients, many studies indicate that dentists' lack knowledge of the etiology and diagnosis of oral cancer (Alfano and Horowitz, 2001). A previous study found that there is a delay in the early detection of oral cancer by the dental professionals (McLeod et al., 2005).

Several studies have assessed the dentists' knowledge, attitudes, and opinions regarding oral cancer and the early detection of oral cancer (Yellowitz et al., 2000; Patton et al., 2005; Gajendra et al., 2006; Ariyawardana and Ekanayake, 2008; Applebaum et al., 2009; Joseph et al., 2012; Alaizari and Al-Maweri, 2014; Saleh et al., 2014; Sarabadani et al., 2016; Mariño et al., 2017; Hashim et al., 2018; Jboor et al., 2019; Khattab et al., 2019). These studies showed the necessity to enhance the knowledge on preventing and early detection of oral cancer. A previous study done in Kuwait among dentists working at the Ministry of Health dental centers and the University dental center assessed their oral cancer awareness (Joseph et al., 2012). Another study, which assessed the oral cancer knowledge among undergraduate dental students in Kuwait University, emphasized the necessity for a structured teaching program with importance on early detection and risk factors of oral cancer (Joseph et al., 2015).

The primary oral health care centers provide dental services in all the six governorates in the State of Kuwait and the dentists working in the primary oral health care centers provide the oral health care for patients. Since there is a lack of baseline information about the oral cancer knowledge levels among the dentists working at the primary oral health care centers in Kuwait, it is important to evaluate their knowledge and practices. The present study was conducted to assess the level of oral cancer knowledge, opinions, attitudes, and practices among dentists working at the primary oral health care centers in Kuwait.

\section{Materials and Methods}

\section{Study design}

This is a cross-sectional study among the dentists practicing at the primary oral health care centers in Kuwait. This study was conducted under the supervision of Kuwait School Oral Health Program in collaboration with the Dental Administration, Ministry of Health, Kuwait. The study protocol was approved by the Dental Administration, Ministry of Health Ethical Committee and the School Oral Health Program Research Committee.

\section{Study setting and participants}

Only dentists working at the primary oral health care centers were included in this study. Six dental assistants distributed the questionnaires; one at each governorate. All the dentists reached were invited to participate in the study. Altogether 410 dentists received the questionnaire.
A covering letter explaining the purpose of the study was included.

\section{Study questionnaire}

A 23-item questionnaire in English was developed and utilized in this study, which included previously validated questions (Patton et al., 2005; Gajendra et al., 2006), and also questions designed for this study. The questionnaire was pretested in a pilot study among 40 dentists who attended the previous annual meeting of Kuwait division of the IADR (KuADR). The first section of the questionnaire included 6 questions regarding dentists' demographic characteristics. The demographic data collected were age, gender, date of graduation, the level of education, nationality, and the working site. The second section included 17 questions about the dentists' oral cancer knowledge, attitude, opinions, and practices. Nine knowledge questions were about the oral cancer risk factors, most common forms of oral cancer, common age of diagnosis, most common sites of oral cancer, symptoms of both early, late stage of oral cancer, and the features of oral cancer metastases. Three questions were about the dentists' opinion on oral cancer training, overall dentists' current knowledge, and mandatory oral cancer screening. One attitude question was regarding attending oral cancer continuing education courses. Four questions were about their current practices including evaluating oral cancer risk factors and managing patients with suspicious lesions.

\section{Statistical analysis}

Data were managed and analyzed using Epi-Info 3.5.3 and SPSS 21.0 software (IBM Corp., Armonk, N.Y., USA). Frequencies and means (SD) were used for data description. Shapiro-Wilks test tested continuous variables for normal distribution. As data were normally distributed, t-test and ANOVA were used to examine the difference in mean knowledge, attitude, and practice scores according to gender, nationality, governorate, and level of education. Pearson correlation coefficient was used to evaluate the correlation between the scores and participants' age and years of experience. The significance level used was $p$ $<0.05$.

\section{Results}

From a total of 410 questionnaires distributed to the dentists, 289 were completed, resulting in a response rate of $71 \%$. Of the 289 dentists who participated, 177 (61\%) were Kuwaiti, 112 (39\%) from other nationalities. The mean age was $35.2 \pm 10.9$ years with the gender distribution of $62.3 \%$ males and $37.7 \%$ females. Seventy-five percent of the dentists had only a bachelor degree, while the remaining quarter had a master degree, MEGD or PhD. Their mean years of experience was $11.7 \pm 11.3$ years. Table 1 summarizes the demographic characteristics of the participants. About $98.9 \%$ of the questions were answered.

The mean (SD; range) knowledge score was 6.58 (1.55; 3-9). Majority of the dentists (73.4\%) answered the knowledge questions correctly. Approximately, all dentists $(99.7 \%)$ were knowledgeable about tobacco use 
Table 1. Demographic Characteristics of the Participating Dentists $(\mathrm{N}=289)$.

\begin{tabular}{lc}
\hline Variable & No. (\%) \\
\hline Gender & $180(62.3)$ \\
Male & $109(37.7)$ \\
Female & $35.2 \pm 10.9$ \\
Age in years (mean \pm SD) & \\
Nationality & $177(61.0)$ \\
Kuwaiti & $112(39.0)$ \\
Non-Kuwaiti (Others) & \\
Governorate & $16(5.5)$ \\
Mubarak Alkabeer & $72(24.9)$ \\
Capital & $69(23.9)$ \\
Hawally & $40(13.8)$ \\
Farwaniya & $44(15.2)$ \\
Jahra & $48(16.6)$ \\
Ahmadi & \\
Level of education & $217(75.1)$ \\
Bachelor degree & $72(24.9)$ \\
More than Bachelor degree & $11.6 \pm 11.3$ \\
Years of experience (mean $\pm \mathrm{SD})$ &
\end{tabular}

and alcohol consumption as the two major oral cancer risk factors. About $83.7 \%$ of the dentists reported the symptoms of late stage of oral cancer correctly, and only one-third (31.3\%) of dentists were aware of the symptoms of the early stage of oral cancer. More than one-third $(37.3 \%)$ of dentists knew the most common age of oral cancer diagnosis. The majority of dentists knew the most common form of oral cancer $(80.6 \%)$, the most common site of oral cancer $(80.3 \%)$ and the most likely lesions associated with oral cancer $(87.9 \%)$. Descriptive analysis of knowledge responses is summarized in Table 2.

Kuwaiti dentists had significantly better mean knowledge scores compared to dentists with other nationalities $(6.87$ vs. $6.19, \mathrm{p}<0.001)$. There was no significant difference in knowledge between male and female dentists $(p=0.07)$, between dentists from different governorates $(p=0.06)$, or between dentists with different level of education $(p=0.61)$. Knowledge scores were significantly correlated with age and years of experiences $(\mathrm{r}=0.271, \mathrm{p}<0.001 ; \mathrm{r}=0.302, \mathrm{p}<0.001$, respectively).

Overall, more than half $(58.5 \%)$ of the dentists answered opinion questions positively with a mean opinion score of $1.74(0.2 ; 0-3)$. About $38 \%$ thought they had adequate training to perform oral cancer screening and only half $(55 \%)$ of them thought that their oral cancer knowledge is current. Most of the dentists (82.4\%) thought that oral cancer screening should be a mandatory procedure at the primary oral health care centers. There was no significant difference in opinion scores based on age, gender, nationality, governorate, the level of education, or years of experience. Opinion responses are summarized in Table 3. The majority of dentists (92.4\%)

Table 2. Descriptive Analyses of Dentists' Knowledge

\begin{tabular}{lcc}
\hline Question & Correct No. (\%) & Incorrect No. (\%) \\
\hline Most common risk factors of oral cancer & $287(99.7)$ & $1(0.3)$ \\
Most likely lesions associated with oral cancer & $254(87.9)$ & $32(11.0)$ \\
Conditions which are associated with fibrosis and reduced mouth opening & $250(86.5)$ & $32(11.0)$ \\
Symptoms of the late stage of oral cancer & $241(83.7)$ & $44(15.3)$ \\
Most common form of oral cancer & $233(80.6)$ & $50(17.3)$ \\
Most common site of oral cancer & $232(80.3)$ & $54(18.7)$ \\
Lymph node characteristics of oral cancer metastases & $214(74.3)$ & $71(25.0)$ \\
Majority of oral cancers diagnosed at age & $106(37.0)$ & $177(61.2)$ \\
Symptoms most commonly expressed in early oral cancer & $90(31.3)$ & $195(67.7)$ \\
\hline
\end{tabular}

Table 3. Descriptive Analyses of Dentists' Opinion and Attitude

\begin{tabular}{lcc}
\hline Question & Yes No. (\%) & No No. (\%) \\
\hline Think patient should have mandatory oral cancer screening & $238(82.4)$ & $48(16.6)$ \\
Knowledge about oral cancer is current & $158(55.0)$ & $129(45.0)$ \\
Adequate training to perform an oral cancer screening & $110(38.0)$ & $176(61.0)$ \\
Interested in continuing education courses regarding oral cancer & $267(92.4)$ & $20(6.9)$ \\
\hline
\end{tabular}

Table 4. Descriptive Analyses of Dentists' Practice

\begin{tabular}{lcccc}
\hline Question & Always No. (\%) & Usually No. (\%) & Sometimes No. (\%) & Never No. (\%) \\
\hline Refer patient with suspicious lesion to a specialist & $162(56.0)$ & $72(25.0)$ & $45(16.0)$ & $8(3.0)$ \\
Review patients' risk factors of oral cancer & $26(9.0)$ & $67(23.0)$ & $166(57.0)$ & $26(9.0)$ \\
Ask patient about tobacco use & $71(25.0)$ & $107(37.0)$ & $89(31.0)$ & $18(6.0)$ \\
Ask patient about alcohol consumption & $12(4.2)$ & $37(13.0)$ & $136(47.0)$ & $101(35.0)$ \\
\hline
\end{tabular}


were interested in attending continuing education courses on oral cancer with a mean score of $0.92(0.27 ; 0-1)$. There was no significant difference in attitude scores based on age, gender, nationality, governorate, the level of education, or years of experience.

The mean practice score was 10.36 (2.19; 5-16). Overall, half (48\%) of dentists always/usually follow the ideal practice regarding screening and managing oral cancer. Approximately, half of the dentists (56\%) would always and a quarter $(25 \%)$ of them usually refer a patient with a suspicious lesion to a specialist. Nearly one-third of the dentists (32\%) reviewed their patients' oral cancer risk factors. Overall, two-third of the dentists $(62 \%)$ reported that they assessed the use of tobacco in their practice. Majority of the dentists working at the primary oral health care centers did not ask their patients about alcohol consumption. There was no significant difference in opinion scores based on age, gender, nationality, governorate, the level of education, or years of experience. Distribution of different practice responses is summarized in Table 4.

\section{Discussion}

Since oral cancer is a disease that it is hard to diagnose in its early stage, dental health care professionals should have a significant role and responsibility in prevention and early detection of oral cancer. Understanding the knowledge, attitudes, and practices among dental practitioners is vital to assess their effectiveness in early detection and to reduce the mortality and morbidity of oral cancer (Horowitz et al., 2000). This study was done to assess the oral cancer knowledge, opinions, attitudes and practices among dentists working at the primary oral health care centers in Kuwait. This study's results are comparable to other studies done among similar participants (Yellowitz et al., 2000; Patton et al., 2005; Gajendra et al., 2006; Applebaum et al., 2009; Joseph et al., 2012; Alaizari and Al-Maweri, 2014; Saleh et al., 2014; Hashim et al., 2018; Jboor et al., 2019; Khattab et al., 2019).

Of 410 questionnaires distributed, 289 dentists returned completed questionnaires with a total response rate of $71 \%$ in this study. This response was higher than in similar studies which had only 9.4\% (Mariño et al., 2017), 27.6\% (Alaizari and Al-Maweri, 2014), 38\% (Ariyawardana and Ekanayake, 2008), 41.7\% (Saleh et al., 2014) and response rates from 50-59\% (Horowitz et al., 2000; Yellowitz et al., 2000; Patton et al., 2005; Gajendra et al., 2006; Applebaum et al., 2009). The reply percentage was similar to a previous study in Kuwait (76.5\%) (Joseph et al., 2012). Less than one-third of dentists did not participate in this study. The possible reason could be that some of the dentists were not confident about their oral cancer knowledge. Another reason could be that some of the dentists graduated from dental schools with Arabic curriculum did not participate as the questionnaire was in English.

Tobacco and alcohol are the most important risk factors for oral cancer (Moreno-Lopez et al., 2000;
Rodriguez et al., 2004; Petersen, 2009). In this study, almost all dentists identified tobacco use and alcohol consumption as the two major risk factors for oral cancer occurrence. This was consistent with recent studies; in which majority of dentists recorded similar oral cancer risk factors (Jboor et al., 2019; Khattab et al., 2019). As shown in prior studies, although a large percentage of dental practitioners identified tobacco and alcohol as major risk factors, few of them assessed their patients for alcohol use (Horowitz et al., 2000; Gajendra et al., 2006).

In the current study, majority of the dentists had a good knowledge on the clinical presentation and risk factors of oral cancer. However, this knowledge could have had higher impact on the dentists' practice. Several studies that had been reviewed showed that dentists were knowledgeable about oral cancer risk factors, signs, and symptoms (Applebaum et al., 2009; Yellowitz et al., 2000; Gajendra et al., 2006; Hashim et al., 2018; Jboor et al., 2019). Also, an earlier study among undergraduate dental students in Kuwait University showed that their knowledge of non-risk factors was lesser than their knowledge of recognized risk factors (Joseph et al., 2015).

In our study, greater number of dental practitioners knew the most common site affected by oral cancer when compared to other studies (Applebaum et al., 2009; Yellowitz et al., 2000; Hashim et al 2018; Jboor et al., 2019). Similar to this study, majority of the dental students identified the most likely sites of oral cancer (Al-Maweri et al., 2015). Compared to this study, higher percentage of dentists in other studies thought that they were adequately trained to perform oral cancer screening (Applebaum et al., 2009; Jboor et al., 2019). Similar percentage of dentists believed that their knowledge regarding oral cancer was current as in our study (Applebaum et al., 2009), however some studies found higher percentage of dentists who perceived that their oral cancer knowledge was up-to-date (Yellowitz et al., 2000; Jboor et al., 2019). In our study, half of the primary oral health care dentists routinely followed the practice regarding screening and managing oral cancer; similar to a recent study in Australia; wherein half of the oral health professionals performed oral cancer screening (Mariño et al,. 2017). In other studies, more than half of the dentists thought that they had learnt the important skills for oral cancer screening (Sarabadani et al., 2016); whereas majority of dentists agreed that they were adequately trained in oral cancer screening (Ariyawardana and Ekanayake, 2008).

An earlier study in Kuwait (Joseph et al., 2012) demonstrated better knowledge of dentists about the symptoms of early oral cancer; wherein majority knew that the early oral cancer is asymptomatic compared to this study. This dissimilarity could be due to the differences between the dentists' affiliations. Majority of dentists routinely referred their patients with suspicious lesions to a specialist in this study which is similar to a previous study in Kuwait (Joseph et al., 2012) and higher than in an earlier study in the US (Patton et al., 2005).

A possible strength in this study was in the sample that only the dentists working at the primary oral health care centers were included. These dentists provide the primary 
oral health care to their patients in their practice and a good response rate was achieved. Another strength of this study was that the questions included in this study were pretested among the dentists who attended the previous KuADR conference in Kuwait. A likely limitation of this study was that dentists practicing in the private clinics were not included.

Delays in the diagnosis and referral of patients with oral cancer have not improved in spite of efforts to increase the awareness of the disease (McLeod et al., 2005). Continuing education courses can have a positive influence on dentists' knowledge and practices (Silverman and Rankin, 2010). Since primary oral health care dentists have a vital role in screening patients for oral cancer, education in oral cancer examination must be a regular part of continuing dental education courses. In this study, almost all dentists were interested in attending continuing education courses regarding oral cancer which is similar to other studies (Applebaum et al., 2009; Hassona et al., 2015; Khattab et al., 2019) and higher than in a previous study that was done in the US, wherein only $50 \%$ of the dentists indicated an interest in further training (Horowitz et al., 2000). Since most of the dentists in this study expressed their interest to attend continuing education training on oral cancer, this might be considered as a strong indicator for improving; as such courses enhance the prevention and early detection of oral cancer. In a recent study, the significance of enhanced educational methods for dentists on oral cancer detection and prevention was emphasized (Kebabcioğlu and Pekiner, 2018).

Since oral cancer is generally linked to lifestyle and as primary oral health care providers; dentists should be aware of the risk factors and have a significant part in imparting knowledge about the benefits from the altering of lifestyle habits (Galvão-Moreira and da Cruz, 2017). Dentists working at the primary oral health care centers play an important role in the early detection of oral cancer and all patients should receive an oral cancer examination which takes only about 90 seconds as a part of a comprehensive oral examination (Horowitz, 2001). One important part of the primary oral health care is the early detection of oral cancer through the visual examination that can be done by the general practitioner dentists with minimal tools. Since, the visual examination of any oral epithelial changes is the gold standard of early detection of oral cancer (Epstein and Lonky, 2008); this examination should be a routine procedure at the primary oral health care centers in Kuwait.

In conclusions majority of the dentists presented good knowledge about various aspects of oral cancer. More continuing education programs on risk factors and diagnosis of oral cancer should be organized to train dentists. Oral cancer screening should be a routine procedure for the high risk patients at the primary oral health care centers in Kuwait.

This study was not funded. There is no conflict of interest regarding the study. Ethical approval for this study was obtained from the Ethical Committee, Dental Administration, Ministry of Health and the School Oral Health Program, Research Committee, Kuwait.

\section{Acknowledgements}

We express special thanks to all the dentists who participated in the study. We gratefully acknowledge the support of the dental assistants, School Oral Health Program, Kuwait for distributing the questionnaire.

\section{References}

Alaizari NA, Al-Maweri SA (2014). Oral cancer: knowledge, practices and opinions of dentists in Yemen. Asian Pac J Cancer Prev, 15, 5627-31.

Alfano MC, Horowitz AM (2001). Professional and community efforts to prevent morbidity and mortality from oral cancer. $J$ Am Dent Assoc, 132, Suppl 24-9.

Al-Maweri SA, Abbas A, Tarakji B, et al (2015). Knowledge and opinions regarding oral cancer among Yemeni dental students. Asian Pac J Cancer Prev, 16, 1765-70.

Applebaum E, Ruhlen T, Kronenberg F, Hayes C, Peters E (2009). Oral cancer knowledge, attitudes and practices: a survey of dentists and primary care physicians in Massachusetts. J Am Dent Assoc, 140, 461-7.

Ariyawardana A, Ekanayake L (2008). Screening for oral cancer/ precancer: knowledge and opinions of dentists employed in the public sector dental services of Sri Lanka. Asian Pac J Cancer Prev, 9, 615-8.

Conway D, Petticrew M, Marlborough H, et al (2008). Socioeconomic inequalities and oral cancer risk: A systematic review and meta-analysis of case-control studies. Int J Cancer, 122, 2811-9.

Epstein JB, Silverman S Jr, Epstein JD, Lonky SA, Bride MA (2008). Analysis of oral lesion biopsies identified and evaluated by visual examination, chemiluminescence and toluidine blue. Oral Oncol, 44, 538-44.

Ferlay J, Shin HR, Bray F, et al (2010). GLOBOCAN 2008, Cancer incidence and mortality wordwide: IARC CancerBase No 10. Lyon, International Agency for Research on Cancer, http://globocan.iarc.fr.

Ferlay J, Soerjomataram I, Ervik M, et al (2013). GLOBOCAN 2012 v1.0, Cancer incidence and mortality worldwide: IARC CancerBase No 11. Lyon, International Agency for Research on Cancer, http://globocan.iarc.fr (accessed 05 May 2016).

Gajendra S, Cruz G, Kumar J (2006). Oral cancer prevention and early detection: knowledge, practices, and opinions of oral health care providers in New York State. J Cancer Educ, 21,157-62.

Galvão-Moreira LV, da Cruz MCFN (2017). Screening and early detection of oral cancer: current controversies. Acta Odontol Scand, 75, 361-5.

Gillison ML (2007). Current topics in the epidemiology of oral cavity and oropharyngeal cancers. Head Neck, 29, 779-92.

Hashim R, Abo-Fanas A, Al-Tak A, Al-Kadri A, Abu Ebaid Y (2018). Early detection of oral cancer- dentists' knowledge and practices in the United Arab Emirates. Asian Pac J Cancer Prev, 19, 2351-5.

Hassona Y, Sawair F, Baqain E, et al (2015). Oral cancer early detection - a pressing need for continuing education in Jordan. Asian Pac J Cancer Prev, 16, 7727-30.

Horowitz AM, Drury TF, Goodman HS, Yellowitz JA (2000). Oral pharyngeal cancer prevention and early detection. Dentists opinions and practices. J Am Dent Assoc, 131, 453-62.

Horowitz AM (2001). Perform a death-defying act. The 90-second oral cancer examination. J Am Dent Assoc, 132, Suppl 36-40.

Jboor DH, Al-Darwish MS, Nur U (2019). Oral cancer 
knowledge, attitude and practice of dentists in the State of Qatar. Dent J, 7, 43.

Joseph BK, Sundaram DB, Sharma P (2012). Oral cancer awareness among dentists in Kuwait. Med Princ Pract, 21, 164-70.

Joseph BK, Sundaram DB, Ellepola AN (2015). Assessing oral cancer knowledge among undergraduate dental students in Kuwait University. J Cancer Educ, 30, 415-20.

Kebabcıŏlu Ö, Pekiner FN (2018). Assessing oral cancer awareness among dentists. $J$ Cancer Educ, 33, 1020-6.

Khattab NMA, Elheeny AAH, Tony GA (2019). Oral-cancer knowledge, practice, and attitude assessment of dentists in Upper Egypt: A cross-sectional study. Clin Exp Dent Res, 10, 1-7.

Kujan O, Farah CS, Johnson NW (2017). Oral and oropharyngeal cancer in the Middle East and North Africa: Incidence, mortality, trends, and gaps in public databases as presented to the Global oral cancer forum. Translational Res in Oral Oncol, 2, 1-9.

Mariño R, Haresaku S, McGrath R, et al (2017). Oral cancer screening practices of oral health professionals in Australia. BMC Oral Health, 17, 151.

McLeod N, Saeed N, Ali E (2005). Oral cancer: Delays in referral and diagnosis persist. Br Dent $J, \mathbf{1 9 8}, 681-4$.

Memon A, Moody PM, Sugathan TN, et al (2000). Epidemiology of smoking among Kuwaiti adults: prevalence, characteristics, and attitudes. Bull World Health Organ, 78, 1306-10.

Messadi D, Wilder-Smith P, Wolinsky L (2009). Improving oral cancer survival: the role of dental providers. J Calif Dent Assoc, 37, 789-98.

Moreno-López L, Esparza-Gómez G, Gonzales-Navarro A, et al (2000). Risk of oral cancer associated with tobacco smoking, alcohol consumption and oral hygiene: a case-control study in Madrid, Spain. Oral Oncol, 36, 170-4.

Morris RE, Mahmeed BE, Gjorgov AN, Jazzaf HG, Rashid BE (2000). The epidemiology of lip, oral cavity and pharyngeal cancers in Kuwait. Br J Oral Maxillofac Surg, 38, 316-9.

Patton LL, Elter JR, Southerland JH, Strauss RP (2005). Knowledge of oral cancer risk factors and diagnostic concepts among North Carolina dentists. Implications for diagnosis and referral. J Am Dent Assoc, 136, 602-10.

Petersen PE (2009). Oral cancer prevention and control - the approach of the World Health Organization. Oral Oncol, 45, 454-60.

Petersen PE (2008). World Health Organization global policy for improvement of oral health - World Health Assembly 2007. Int Dent J, 58, 115-21.

Rodriguez T, Altieri A, Chatenoud L, et al (2004). Risk factors for oral and pharyngeal cancer in young adults. Oral Oncol, 40, 207-13.

Saleh A, Kong YH, Vengu N, et al (2014). Dentists' perception of the role they play in early detection of oral cancer. Asian Pac J Cancer Prev, 15, 229-37.

Sarabadani J, Pakfetrat A, Dalirsani Z, Motezarre HR (2016). Oral cancer: prevention and early detection, dentists' opinions and practices (Mashhad-Iran). Int J High Risk Behav Addict, 5, e30987.

Silverman S Jr, Rankin KV (2010). Oral and pharyngeal cancer control through continuing education. J Cancer Educ, 25, 277-8.

Thun MJ, DeLancey JO, Center MM, Jemal A, Ward EM (2010). The global burden of cancer: priorities for prevention. Carcinogenesis, 31, 100-10.

Warnakulasuriya S (2009). Global epidemiology of oral and oropharygeal cancer. Oral Oncol, 45, 309-16.

Yellowitz JA, Horowitz AM, Drury TF, Goodman HS (2000). Survey of U.S. dentists' knowledge and opinions about oral pharyngeal cancer. J Am Dent Assoc, 131, 653-61.

\section{(ब) $(\mathbb{Q} \otimes$}

This work is licensed under a Creative Commons AttributionNon Commercial 4.0 International License. 